\title{
Non-Small Cell Lung Cancer Patients Harboring HER2 Mutations: Clinical Characteristics and Management in a Real-Life Setting. Cohort HER2 EXPLORE GFPC 02-14
}

Jean-Bernard Auliac · Pascal Dô · Sophie Bayle · Hélène Doubre ·

Florent Vinas · Jacques Letreut • Lionel Falchero • Pierre Alexandre Hauss •

Pascal Thomas · Christos Chouaid (D)

Received: April 13, 2019 / Published online: June 1, 2019

(c) The Author(s) 2019

\section{ABSTRACT}

Background: Mutation of human receptor tyrosine kinase epidermal growth factor receptor-2 (HER2) is a rare event, found in approximately $1 \%$ non-small cell lung cancers (NSCLC). The objective was to investigate the clinical characteristics and management of HER2-mutated NSCLCs in a real-life setting.

Methods: This multicenter study described NSCLCs harboring HER2 mutations diagnosed between January 2012 and December 2014, according their clinical characteristics,

Enhanced digital features To view enhanced digital features for this article go to https://doi.org/10.6084/ m9.figshare. 8138837 .

J.-B. Auliac

Chest Department, Hopital de Mantes La Jolie,

Mantes-la-Jolie, France

P. Dô

Oncolgy Department, Centre Francois Baclesse,

Caen, France

S. Bayle

Chest Department, Institut de Cancérologie,

Saint-Priest-en-Jarez, France

H. Doubre

Chest Department, Hôpital Foch, Suresnes, France

F. Vinas · C. Chouaid $(\bowtie)$

Chest Department, Centre Hospitalier

Intercommunal de Créteil, Créteil, France

e-mail: christos.chouaid@chicreteil.fr management, and outcomes: response rate (RR), progression-free survival (PFS), and overall survival (OS).

Results: Thirty patients were included: $66.7 \%$ women; median age $65.2 \pm 12$ years; never or former smokers $73.3 \%$. Of the stage IV patients $(n=23), 86 \%$ received first-line platin doublet chemotherapy: RR $61.5 \%$ and PFS 6.7 (95\% CI 5.9-9.5) months; $52.1 \%$ received a second-line therapy: RR $18.2 \%$ and PFS 4.9 (95\% CI 2.5-11.9) months. Median OS of stage IV was 10.7 months and 2-year OS was $27.2 \%$ (95\% CI 11.7-63.2). All patients with stage I-III NSCLCs were alive at 2 years.

Conclusion: The rarity of HER2-mutated NSCLCs requires specific studies.

\section{J. Letreut}

Chest Department, Hopital d'Aix-en-Provence,

Aix-en-Provence, France

L. Falchero

Chest Department, Hopital de Villefranche, Villefranche, France

\section{P. A. Hauss}

Chest Department, Hopital D’Elbeuf, Elbeuf, France

P. Thomas

Chest Department, Hopital de Gap, Gap, France 
Keywords: HER2 mutation; Lung adenocarcinoma; Management; Prognosis; Target therapy

\section{INTRODUCTION}

Human receptor tyrosine kinase epidermal growth-factor receptor-2 (human epidermal growth factor receptor-2; HER2) is a membranebound ErbB family tyrosine kinase. No ligand has been described for this receptor, which is activated by homodimerization or heterodimerization with other ErbB family members [1-3]. The HER2 gene has been a recognized proto-oncogene in human cancers for more than two decades. All of the mutations identified were inframe, 3-12 base-pair insertions into exon 20, leading to constitutive activation of the receptor, and downstream AKT (protein kinase B) and MEK [mitogen-activated protein kinase (MAPK)-extracellular signal-regulated kinase (ERK)] pathways. The ErbB2 gene, which encodes HER2, is a major proliferation driver that activates downstream signaling through phosphoinositide-3kinase (PI3K)-AKT and MEK-ERK pathways [1-3]. HER2 mutations satisfy the genetic driver definition and preclinical models proved the concept of the transforming ability of such a genetic alteration. Inducing mutant HER2 expression in the lung epithelium of mice results in the emergence of invasive adenosquamous carcinomas, with tumor maintenance requiring continuous driver expression, as observed with epidermal growth factor receptor (EGFR)-driven cancers [3].

In non-small cell lung cancers (NSCLCs), HER2 mutation is a rare event [1, 4-7], occurring in approximately $1 \%$ of the overall lung cancer population. This rate increases to $4.8 \%$ in $E G F R$ wild-type lung adenocarcinoma resection specimens [1] and 5.1\% in EGFR-/KRAS(Kirsten rat sarcoma viral oncogene)/BRAF- (vRaf murine sarcoma viral oncogene homolog B)/ALK- (anaplastic lymphoma kinase)/ROS1 (ROS proto-oncogene-1, receptor tyrosine kinase)-negative patients [8]. The clinical phenotype resembles that of patients with EGFRmutated tumors [9]. HER2 involvement in lung carcinogenesis has been known for many years but clinical research was slowed by the negative results obtained with trastuzumab in the first clinical trial. Indeed, adding trastuzumab to gemcitabine-cisplatin or docetaxel failed to show any survival benefit for patients with HER2 immunohistochemistry (IHC)-positive lung cancers [10].

This retrospective multicenter study was undertaken to improve our understanding of HER2-mutated NSCLCs by analyzing the clinical-pathological characteristics of patients with NSCLCs harboring the HER2 mutation.

\section{METHODS}

Physicians at French medical centers were asked to provide retrospectively anonymized data from the medical records of patients at least 18 years old when first diagnosed with HER2mutated NSCLCs between January 2012 and December 2014. HER2 mutations were identified via one of the following methods: (1) multiplexed sizing assays for insertions and deletions in EGFR and ErB2; (2) mutational hotspot testing by a mass spectrometry-based nucleic acid assay on the Sequenom ${ }^{\mathrm{TM}}$ platform for 91 mutations in six genes (EGFR, HER2, KRAS, NRAS, BRAF, and PIK3CA). Patient demographics and clinical characteristics at NSCLC diagnosis, including age, sex, smoking history (never smoked, current smoker, former smoker), cancer histology, and presence of metastases were collected from patients' charts; treatment information from diagnosis included treatment sequencing and types; clinical outcomes included dates of clinician-defined progression-free survival (PFS) for first-line and second-line treatments based on increased lesion size(s), appearance of new lesions, or clinical worsening and death (if applicable). Overall survival (OS) from diagnosis to death was recorded.

Patient characteristics and treatment information were analyzed descriptively. To evaluate OS, patients were censored at the last follow-up visit. To analyze PFS, patients who died were considered to have progressed. All analyses were computed with SAS v9.3 software (SAS Institute Inc., Cary, NC, USA). 
The study was granted the institutional review board (IRB) approval of Saint Etienne (IRBN 102,016/Chuste) and had Comité Independant de Traitement des Ressources de Santé (CITRS) authorization $(914,146)$. According to French legislation, information was provided orally to each included patient. The study complied with the International Ethical Guidelines for Biomedical Research Involving Human Subjects, Good Clinical Practice Guidelines, and local laws. It was also performed in accordance with the Helsinki Declaration of 1964 and its later amendments.

\section{RESULTS}

Physicians from 17 medical centers extracted information on 30 NSCLC patients (Table 1). At diagnosis, median age was $65.2 \pm 12$ years; $66.7 \%$ were women; $90 \%$ had good performance status (PS 0-1); 30\% and 43.3\% were never or former smokers, respectively; $93.3 \%$ had adenocarcinomas, with $76.7 \%$ having advanced disease at diagnosis; $20 \%$ had a previous cancer(s) and 8\% a family history of cancer. All tumors had an in-frame insertion within the HER2 gene exon 20 coding sequence. Most of the mutations were exclusive, except for one patient with concomitant $A L K$ translocation and another with RET (REarranged during

Table 1 HER2-mutated NSCLC patient characteristics at diagnosis

\begin{tabular}{lll}
\hline Characteristic & $\begin{array}{l}\text { All stages } \\
(\boldsymbol{n}=\mathbf{3 0})\end{array}$ & $\begin{array}{l}\text { Stage IV } \\
(\boldsymbol{n}=\mathbf{2 3})\end{array}$ \\
\hline Age (years) & $65.2 \pm 12$ & $64.4 \pm 13$ \\
Women & $20(66.7 \%)$ & $15(65.2 \%)$ \\
Never & $9(30 \%)$ & $7(30.4 \%)$ \\
Former smokers & $13(43.3 \%)$ & $10(43.4 \%)$ \\
Performance status $0-1$ & $27(90 \%)$ & $19(82.6 \%)$ \\
Weight loss < 5\% & $27(90 \%)$ & $21(91.3 \%)$ \\
Caucasian & $26(86.7)$ & $21(91.3 \%)$ \\
Adenocarcinoma & $28(93.3 \%)$ & $22(95.7 \%)$ \\
Symptomatic patients & $27(90)$ & $20(87 \%)$ \\
\hline
\end{tabular}

Transfection) translocation. The majority of patients $(93.1 \%)$ were symptomatic at diagnosis, with the main symptoms being respiratory $(77.8 \%)$, pain $(25.9 \%)$, or neurological $(11.1 \%)$. The most frequent metastatic sites were lung $(38.1 \%)$, brain $(19 \%)$, liver $(14.3 \%)$, and $36.3 \%$ had more than two metastatic lesions.

Among the 23 stage IV NSCLC patients, $86 \%$ received first-line chemotherapy with platinbased doublets and bevacizumab (14\% received best supportive care); none was prescribed antiHER2 therapy. First-line therapy response rate (RR), disease control rate (DCR), and PFS were $61.5 \%, 84.6 \%$, and 6.7 (95\% confidence interval (CI) 5.9-9.5) months, respectively. Progression was characterized by increased sizes of existing lesions $(85.7 \%)$ and/or appearance of new lesions $(64.3 \%)$, most often in the lungs $(55.6 \%)$ or bones $(11.1 \%)$. New biopsies were obtained from three patients at the first progression but had no impact on NSCLC management. Among the 14 patients who discontinued first-line chemotherapy, $85.7 \%$ received second-line therapy, mainly docetaxel, achieving respective RR, DCR, and PFS of $18.2 \%, 36.4 \%$, and 4.9 (95\% CI 2.5-11.9) months. For stage IV patients, 2-year OS was $27.2 \%$ (95\% CI 11.7-63.2\%) and median OS lasted 10.7 months. All seven stage I-III NSCLC patients were alive at 2 years.

\section{DISCUSSION}

The clinical and molecular characteristics of HER2-mutated NSCLCs included in this study are consistent with other series. As in our cohort, HER2 mutations appear more common in younger patients, female patients, nonsmokers, and adenocarcinomas [8]. In a retrospective study on 64 patients with HER2-mutated NSCLCs [11], median age was 62 years, with higher percentages of women $(63 \%)$ and never smokers (58\%); all tumors were adenocarcinomas. In a European cohort [6], median age was 61 years, $62.4 \%$ were women, and $60.4 \%$ had never smoked. The same characteristics were found in an Asian analysis [8].

Usually, previously described patients had good performance status, allowing a majority to be treated with chemotherapy, $86 \%$ herein and 
$79 \%$ in the study by Mazières et al. [6]. Chemotherapy responses also seem to have been better than for non-selected NSCLC patients: RR $61.5 \%$ and PFS of 6.7 months for our cohort and $43.5 \%$ and 6 months, respectively, for the European cohort [6]. A high percentage of patients also received second-line therapy: $85.7 \%$ herein and $77 \%$ in the European analysis, with PFS exceeding 4 months in both studies. Those results are clearly better that those of patients who received second-line therapy for their NSCLCs without oncogene mutation(s) [11]. This more favorable outcome of patients with HER2mutated NSCLCs, seen herein and as reported by others, might be explained by patient selection, i.e., mainly women and non-smokers, rather than the potential prognostic value of HER2 mutation. However, in a large cohort analysis [12], median OS (19 months) of the HER2-mutated NSCLC patients did not differ significantly from that of other molecularly defined cohorts.

Although HER2 mutation appears to be an emerging and promising drug-targetable NSCLC marker, the optimal choice of targeted therapy remains poorly defined. Several phase I/II trials [13-15] are investigating the efficacy of irreversible pan-ERBB receptor family inhibitors, such as dacomitinib, neratinib, and pyrotinib. The National Comprehensive Cancer Network (NCCN) recommended trastuzumab or afatinib as potential therapy options for NSCLC patients with HER2 mutations [16]. In the European cohort, patients treated with HER2-targeting agents had a 5.1-month PFS from the start of the first HER2-specific treatment. Dacomitinib, an irreversible inhibitor of HER1, HER2, and HER4 tyrosine kinases, achieved partial responses in $12 \%$ of patients with HER 2 mutations; afatinib afforded modest responses in $18.2 \%$ and median PFS lasted 3.9 months [6].

In a retrospective study [11], $61 \%$ of 38 patients received one or more HER2-targeted therapies (dacomitinib, afatinib, neratinib, and lapatinib), the median duration of HER2 tyrosine kinase inhibitors was 2.2 months (28 treatments, range $0.3-16.3$ months), and durable clinical benefit of HER2-targeted agents was only seen in some patients, including two given dacomitinib for 13 and 17 months, and one prescribed lapatinib for 10 months.
Specific anti-HER2 treatment outcomes were disappointing in a phase II trial of dacomitinib for patients with HER2-mutated lung cancers; none of the 13 patients responded [13]. A recent phase II study investigated pyrotinib, a novel EGFR/HER2 inhibitor, in heavily pretreated patients with HER2-mutated adenocarcinomas obtained promising results: $54.5 \%(6 / 11)$ RR and median PFS lasted 6.2 months [15]. Poziotinib appears also to have some promising activity in this setting in preliminary results [17]. Large cohort studies are still needed to validate pyrotinib efficacy in this setting. The molecular complexities may explain why not all HER2 mutations or HER2-targeting agents are the same and, for reasons not yet discovered, only a subset of such patients responds to various targeted agents. Chemotherapy, particularly pemetrexed doublets, remains the standard of care for these patients.

Our retrospective analysis was conducted to evaluate real-life data for patients with HER2mutated NSCLCs. It has several limitations. First, because it was a retrospective and observational study, collected patient information was not always complete. Although the data reflect clinical practices, it is important to note that, given the study inclusion period, the results cannot be generalized to current practice with more therapeutic options now available. Another limit is that the type of HER2 mutant variant was not assessed in the majority of cases. However, because of the rarity of HER2-mutated NSCLCs and the number of participating centers, we think that this analysis provides valuable real-life information about treatments received and outcomes of patients with HER2mutated NSCLCs.

\section{CONCLUSION}

HER2 mutations are rare in NSCLCs and appear to be more common in younger patients, female patients, non-smokers, and adenocarcinoma histology. In these patients, NSCLC responses to platin chemotherapy seem to have been better than for non-selected NSCLCs and this remains the standard of care as no target therapy has yet achieved satisfactory outcomes. 


\section{ACKNOWLEDGEMENTS}

We thank the participants of the study.

Funding. This study was supported by academic grants from Lilly, Astra Zeneca, and Boehringer Ingelheim Laboratories. The article processing charges and open access fee were funded by the Groupe Français de Pneumo-Oncologie (GFPC), an academic organization. The sponsors had no role in the design or performance of the study, data analysis, or manuscript preparation. The data were analyzed by the GFPC statistician and interpreted by the authors.

Authorship. All authors meet the International Committee of Medical Journal Editors (ICMJE) criteria for authorship for this article, take responsibility for the integrity of the work as a whole, and have given their approval for this version to be published.

Prior Presentation. Preliminary results of this study were presented in an abstract at the World Conference on Lung Cancer 2016, Vienna.

Disclosures. The Jean-Bernard Auliac, Pascal Dô, Sophie Bayle, Hélène Doubre, Florent Vinas, Jacques Letreut, Lionel Falchero, Pierre-Alexandre Hauss, Pascal Thomas, and Christos Chouaid declare no conflicts of interest with the subject of this manuscript.

Compliance with Ethics Guidelines. The study was granted the IRB approval of Saint Etienne (IRBN 102016/Chuste) and had Comité Independant de Traitement des Ressources de Santé (CITRS) authorization $(914,146)$. According to French legislation, information was provided orally to each included patient. The study complied with the International Ethical Guidelines for Biomedical Research Involving Human Subjects, Good Clinical Practice Guidelines, and local laws. It was also performed in accordance with the Helsinki Declaration of 1964 and its later amendments.

Data Availability. All data generated or analyzed during this study are included in this published article.
Open Access. This article is distributed under the terms of the Creative Commons Attribution-NonCommercial 4.0 International License (http://creativecommons.org/licenses/ by-nc/4.0/), which permits any noncommercial use, distribution, and reproduction in any medium, provided you give appropriate credit to the original author(s) and the source, provide a link to the Creative Commons license, and indicate if changes were made.

\section{REFERENCES}

1. Li BT, Ross DS, Aisner DL, et al. HER2 amplification and HER2 mutation are distinct molecular targets in lung cancers. J Thorac Oncol. 2016;11:414-9.

2. Chan BA, Hughes BG. Targeted therapy for nonsmall cell lung cancer: current standards and the promise of the future. Transl Lung Cancer Res. 2015;4:36-54.

3. Ou SI, Schrock AB, Bocharov EV, et al. HER2 transmembrane domain (TMD) mutations (V659/ G660) that stabilize homo- and heterodimerization are rare oncogenic drivers in lung adenocarcinoma that respond to afatinib. $\mathrm{J}$ Thorac Oncol. 2017;12:446-57.

4. Mazières J, Peters S, Lepage B, et al. Lung cancer that harbors an HER2 mutation: epidemiologic characteristics and therapeutic perspectives. J Clin Oncol. 2013;31:1997-2003.

5. Barlesi F, Mazières J, Merlio JP, et al. Routine molecular profiling of patients with advanced nonsmall-cell lung cancer: results of a 1-year nationwide programme of the French Cooperative Thoracic Intergroup (IFCT). Lancet. 2016;387:1415-26.

6. Mazières J, Barlesi F, Filleron $\mathrm{T}$, et al. Lung cancer patients with HER2 mutations treated with chemotherapy and HER2-targeted drugs: results from the European EUHER2 cohort. Ann Oncol. 2016;27:281-6.

7. Kris MG, Johnson BE, Kwiatkowski DJ, et al. Identification of driver mutations in tumor specimens from 1,000 patients with lung adenocarcinoma: The NCI's Lung Cancer Mutation Consortium (LCMC). J Clin Oncol. 2011;29 (abstr CRA7506).

8. Li X, Zhao C, Su C, Ren S, Chen X, Zhou C. Epidemiological study of HER-2 mutations among EGFR wild-type lung adenocarcinoma patients in China. BMC Cancer. 2016;16:828. 
9. De Grève J, Teugels $\mathrm{E}$, Geers $\mathrm{C}$, et al. Clinical activity of afatinib (BIBW 2992) in patients with lung adenocarcinoma with mutations in the kinase domain of HER2/neu. Lung Cancer. 2012;76:123-7.

10. Gatzemeier U, Groth G, Butts C, van Zandwijk N. Randomized phase II trial of gemcitabine-cisplatin with or without trastuzumab in HER2-positive nonsmall-cell lung cancer. Ann Oncol. 2004;15:19-27.

11. Eng J, Hsu M, Chaft JE, et al. Outcomes of chemotherapies and HER2 directed therapies in advanced HER2-mutant lung cancers. Lung Cancer. 2016;99:53-6.

12. Arcila ME, Chaft JE, Nafa K, et al. Prevalence, clinicopathologic associations, and molecular spectrum of ERBB2 (HER2) tyrosine kinase mutations in lung adenocarcinomas. Clin Cancer Res. 2012; 18:4910-8.

13. Kris MG, Camidge DR, Giaccone G, et al. Targeting HER2 aberrations as actionable drivers in lung cancers: phase II trial of the pan-HER tyrosine kinase inhibitor dacomitinib in patients with HER2mutant or amplified tumors. Ann Oncol. 2015;26:1421-7.

14. Besse B, Soria J-C, Yao B. Neratinib with or without temsirolimus in patients with non-small cell lung cancer carrying HER2 somatic mutations: an international randomized phase II study. ESMO Congress 2014. Abstract LBA39 PR.

15. Ren S, Gao G, Wu F, et al. Preliminary results of a phase II study about the efficacy and safety of pyrotinib in patients with HER2 mutant advanced NSCLC. WCLC 2016. Abstract MA 0403.

16. National Comprehensive Cancer Network. Nonsmall cell lung cancer (Version 3.2016); 2016. https://www.nccn.org/professionals/physician_gls/ pdf/nscl.pdf. Accessed 1 Jan 2019.

17. Oh IJ, Hur JY, Park CK, et al. Clinical activity of PanHER inhibitors against HER2-mutant lung adenocarcinoma. Clin Lung Cancer. 2018;19:e775e78181. 